\title{
Metabolic reprogramming in macrophages and dendritic cells in innate immunity
}

\author{
Beth Kelly ${ }^{1}$, Luke AJ O’Neill ${ }^{1}$ \\ ${ }^{I}$ School of Biochemistry and Immunology, Trinity Biomedical Sciences Institute, Trinity College Dublin, Ireland
}

\begin{abstract}
Activation of macrophages and dendritic cells (DCs) by pro-inflammatory stimuli causes them to undergo a metabolic switch towards glycolysis and away from oxidative phosphorylation (OXPHOS), similar to the Warburg effect in tumors. However, it is only recently that the mechanisms responsible for this metabolic reprogramming have been elucidated in more detail. The transcription factor hypoxia-inducible factor-1 $\alpha$ (HIF-1 $\alpha$ ) plays an important role under conditions of both hypoxia and normoxia. The withdrawal of citrate from the tricarboxylic acid (TCA) cycle has been shown to be critical for lipid biosynthesis in both macrophages and DCs. Interference with this process actually abolishes the ability of DCs to activate T cells. Another TCA cycle intermediate, succinate, activates HIF-1 $\alpha$ and promotes inflammatory gene expression. These new insights are providing us with a deeper understanding of the role of metabolic reprogramming in innate immunity.
\end{abstract}

Keywords: macrophage; dendritic cell; metabolic reprogramming; glycolysis; oxidative phosphorylation Cell Research (2015) 25:771-784. doi:10.1038/cr.2015.68; published online 5 June 2015

\section{Introduction}

Macrophages and dendritic cells (DCs) are the frontline cells of innate immunity. They sense and immediately respond to invading pathogens, thus providing an early defense against external attack. Pattern recognition receptors (PRRs) on the surfaces of these cells recognize pathogen-associated molecular patterns (PAMPs) present in the invaders and, as a result, activate intracellular signaling cascades, leading to the induction of a general pro-inflammatory response [1]. This includes the release of antimicrobial mediators, which target the invading pathogen, chemokines, which recruit more immune cells to the site of infection, and pro-inflammatory cytokines, which drive further inflammation, and induce the adaptive immune response, which is mediated by $\mathrm{T}$ and $\mathrm{B}$ lymphocytes and is more specific for the particular invading pathogen. PRRs are also capable of detecting endogenous danger-associated molecular patterns (DAMPs) [2], which indicate damage to host cells. There are several families of PRRs, the best characterized being the toll-like receptors (TLRs) and the NOD-like receptors (NLRs).

Correspondence: Beth Kelly ${ }^{\mathrm{a}}$, Luke AJ O’Neill ${ }^{\mathrm{b}}$

aE-mail: kellyb3@tcd.ie

bE-mail: laoneill@tcd.ie
The key output of PRRs is increased gene expression. However, cells activated by PAMPs also undergo profound metabolic changes. These changes are required for biosynthesis and energy production, and in addition are involved in signaling processes. Here, we describe recent findings in the field of metabolic reprogramming in innate immunity and place them in the context of our current understanding of the complex events occurring during innate immune cell activation.

\section{Early work on metabolism in innate immune cells}

The Warburg effect is an important concept for understanding metabolic changes occurring in innate immune cells upon activation [3]. Otto Warburg described a metabolic profile of tumors in normoxic conditions, in which glycolysis predominates even though there is oxygen available for oxidative metabolism to proceed. Pyruvate that is produced by the glycolytic pathway, instead of feeding into the tricarboxylic acid (TCA) cycle and subsequent oxidative phosphorylation (OXPHOS), is metabolized to lactate. The Warburg Effect is depicted in Figure 1.

This concept reappeared, but in the innate immune world, in the 1950s, with the discovery that neutrophils depend on aerobic glycolysis for ATP production, and have few mitochondria [4]. Glucose consumption in 
these cells was high, while oxygen consumption was low. Activation of TLR4 by the Gram-negative bacterial product lipopolysaccharide (LPS) in neutrophils increases glucose consumption. It also increases oxygen consumption, but this oxygen is used to produce the reactive oxygen species $\mathrm{H}_{2} \mathrm{O}_{2}$, which is important for neutrophil-mediated killing [5]. Increased flux through the pentose phosphate pathway (PPP), which branches off glycolysis, generates nicotinamide adenine dinucleotide phosphate (NADPH), which is used by NADPH oxidase to generate $\mathrm{H}_{2} \mathrm{O}_{2}[6]$.

In 1969, Hard [7] observed that activated macrophages exhibited increased glycolysis, accompanied by decreased oxygen consumption. Almost 20 years later,

(A) Normal Differentiated Cell, Quiescent Cell

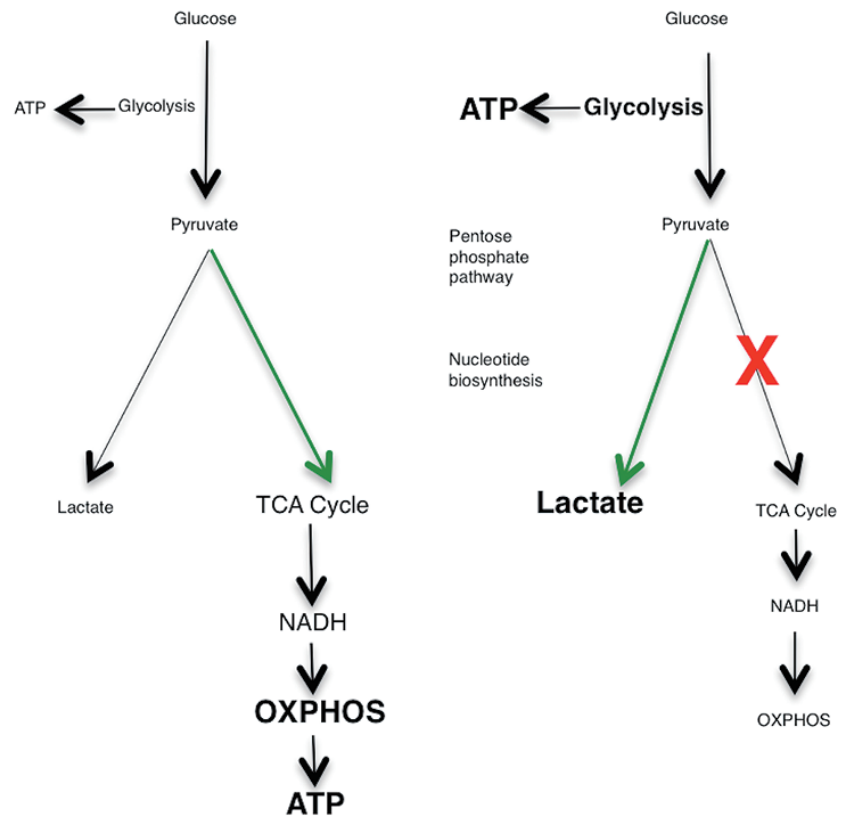

Figure 1 The Warburg effect. (A) In resting cells, glucose is metabolized to pyruvate via glycolysis. Some pyruvate is converted to lactate, but most is directed to the TCA cycle via acetyl-CoA. The TCA cycle generates NADH, which donates electrons to the mitochondrial electron transport chain so that OXPHOS can progress. (B) In highly proliferative or tumour cells, the metabolic profile switches from OXPHOS to aerobic glycolysis, known as the Warburg effect. Mature innate immune cells also rely on glycolysis, although they do not proliferate after activation. The majority of the pyruvate generated by glycolysis is converted to lactate, and glycolytic intermediates build up, meeting the high energy demand of the cell. Glycolysis is the source of ATP in these cells, and also provides glucose-6-phosphate for nucleotide biosynthesis in the PPP.
Newsholme et al. [8] investigated macrophage metabolism and showed that most of the glucose consumed by resting macrophages was converted to lactate, with little being used for OXPHOS. Activated macrophages were shown to have increased expression of the glycolytic enzymes hexokinase and glucose-6-phosphate dehydrogenase, indicating an increase in glycolytic activity in these cells [9].

However, despite these early observations about the metabolism of innate immune cells, it is only more recently that we have begun to elucidate the mechanisms behind these metabolic changes, and appreciate their impact on immune function.

\section{How does LPS promote Warburg metabolism in macrophages and DCs?}

Activation of macrophages or DCs with a range of stimuli, including LPS [10], the TLR3 ligand poly(I:C) [11], and type I interferon (IFN) [11], induces a metabolic switch from OXPHOS to glycolysis, in a phenomenon similar to the Warburg effect [10]. TCA cycle activity is decreased $[10,12]$, while lactate production and flux through the PPP are increased [13, 14]. Enhanced PPP activity boosts production of purines and pyrimidines, which can then be used for biosynthesis in the activated cell. It also provides NADPH for the NADPH oxidase enzyme, which produces ROS [15]. This metabolic shift occurs in mature activated immune cells even though they are not highly proliferative. The increase in glycolysis could be a mechanism to rapidly generate ATP. It is an inefficient means of generating ATP when compared to the TCA cycle, but it can be quickly induced and could be a particularly useful means of synthesizing ATP when cellular glucose uptake capacity is high [16]. Furthermore, in activated macrophages, mitochondrial ROS production is increased, serving as a mechanism of bacterial killing [17]. Glycolytic ATP production may compensate for this shift in mitochondrial metabolism away from ATP production and towards ROS production by the electron transport chain [18].

Substantial insights have been made into the underlying mechanisms of this metabolic switch. There are at least four main processes leading to Warburg metabolism in LPS-activated macrophages and DCs (Figure 2). As the majority of studies investigating this mechanism use LPS as a stimulus, this review focuses on the metabolic alterations induced by LPS in macrophages and DCs. Other innate immune cell stimuli also alter metabolic parameters in innate immune cells, including IFN $\gamma$ [11], poly(I:C) [11], and infection with bacterial species such as Listeria monocytogenes, Mycobacterium bovis BCG 
[19], and Salmonella typhimurium [17].

\section{NO and metabolic changes in macrophages and DCs}

Stimulation of macrophages [20] and DCs [21] with LPS and IFN- $\gamma$ increases the expression of inducible nitric oxide synthase (iNOS), which generates nitric oxide (NO), a reactive nitrogen species that can inhibit mitochondrial respiration. NO achieves this by nitrosylating iron-sulfur proteins present in electron transport chain complexes, e.g., Complex I $[22,23]$, as well as cytochrome c oxidase [24]. The metabolic switch in macrophages and DCs activated by LPS is therefore dependent on iNOS and NO-mediated inhibition of mitochondrial metabolism. Inhibition of iNOS after LPS stimulation restores normal mitochondrial respiration, and treatment of iNOS-deficient DCs with LPS fails to shut down mitochondrial metabolism. Simultaneously, the LPS-induced increase in glycolysis in these cells is blunted. Convincingly, re-addition of $\mathrm{NO}$, using an exogenous $\mathrm{NO}$ donor, to DCs in which iNOS is still inhibited by a specific inhibitor again induces mitochondrial dysfunction and the switch towards glycolytic metabolism. This suggests that iNOS-generated NO is the mediator of the LPS-induced mitochondrial functional collapse and increase in glycolysis [25]. Tumor necrosis factor (TNF)- $\alpha$-induced protein 8-like 2 (TIPE2) is a recently described factor negatively regulating LPS-induced iNOS expression and NO production in macrophages [26].

The amino acid arginine is the substrate of iNOS. In macrophages activated by LPS and IFN- $\gamma$ or bacterial infection (e.g., with M. bovis BCG), extracellular arginine is imported into the cell and is metabolized to NO and citrulline by iNOS. The produced citrulline is then exported from the cell. When extracellular arginine is depleted, citrulline can be imported into the cell and recycled to arginine via argininosuccinate synthase (Ass1) and argininosuccinate lyase (Asl) [19]. This optimum NO production sustained by citrulline recycling via Ass 1 and Asl may be important for controlling mycobacterial infections, as Ass1-deficient mice were less well able to control M. tuberculosis infection [19]. In fact, some pathogens themselves have mechanisms for depleting arginine. For example, Helicobacter pylori expresses an arginase that inhibits NO production by activated macrophages, leading to less effective bacterial killing by these macrophages [27].

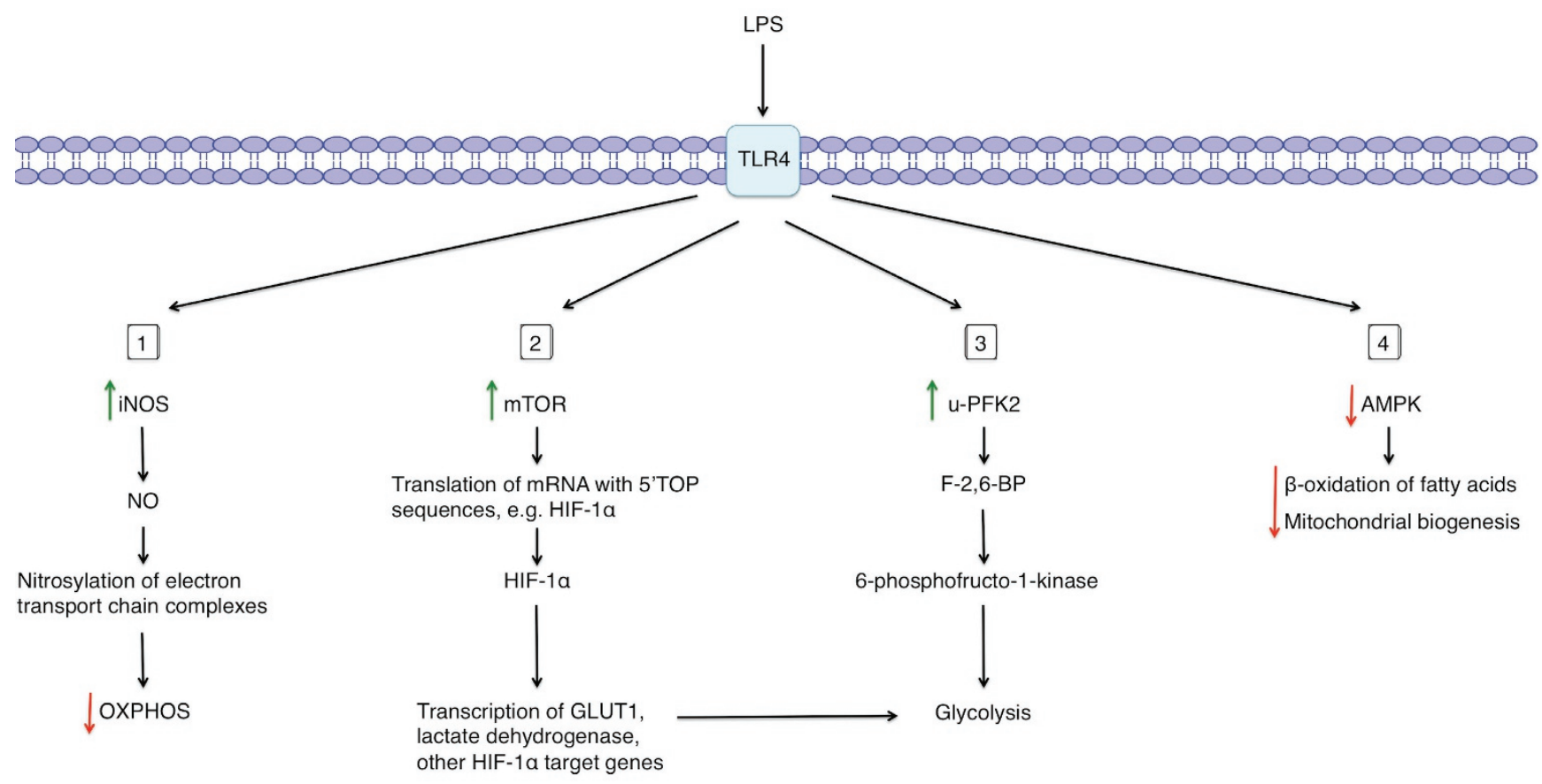

Figure 2 Mechanisms of LPS-induced Warburg metabolism in macrophages or DCs. Upon LPS stimulation of TLR4, a range of metabolic changes occur in macrophages or DCs. (1) LPS activation upregulates iNOS expression, increasing the production of NO, which nitrosylates and thus inhibits target proteins in the mitochondrial electron transport chain, thereby dampening OXPHOS. (2) LPS activates mTOR, thereby increasing the translation of mRNA with 5'-TOP sequences, including HIF-1 $\alpha$ mRNA. HIF-1 $\alpha$ then increases expression of its target genes. (3) LPS increases expression of u-PFK2, an isoform of PFK2, thereby increasing levels of the metabolite F-2,6-BP. F-2,6-BP activates the glycolytic enzyme 6-phosphofructo-1-kinase. (4) Finally, LPS inhibits AMPK, resulting in decreased $\beta$-oxidation of fatty acids and mitochondrial biogenesis. 
A previous study used mass spectrometry to identify nitrosylated proteins in various mouse tissues and revealed that many metabolic enzymes are S-nitrosylated on cysteine residues by NO, including enzymes involved in glycolysis, the TCA cycle and fatty acid metabolism [28]. It is likely that cysteine nitrosylation could affect the activity of these enzymes. Indeed, nitrosylation of the liver enzyme very long-chain acyl-CoA dehydrogenase (VLCAD) was reported to increase its activity, thereby boosting fatty acid metabolism, as VLCAD catalyzes the first step in $\beta$-oxidation of fatty acids [28]. It remains to be investigated whether nitrosylation of these metabolic enzymes is involved in the metabolic switch in activated innate immune cells.

In summary, NO is a key intermediate in the metabolic switch of activated immune cells, and nitrosylation of cellular targets is an important mechanism by which NO exerts its effects.

\section{Hypoxia-inducible factor-1 $\alpha(H I F-1 \alpha)$ and glycolysis}

In tumors, many cells are often exposed to hypoxic microenvironments where they cannot rely on OXPHOS and must modify their metabolism to survive in these conditions of reduced oxygen tension. The transcription factor HIF-1 $\alpha$ [29] promotes the switch to glycolysis so that these cells can continue to produce ATP when oxygen is limited, as oxygen is not required for glycolysis. In such situations of anaerobic glycolysis, pyruvate, the end product of glycolysis, does not feed into the TCA cycle to boost subsequent OXPHOS, but is instead metabolized to lactate. Thus, a hallmark of anaerobic glycolysis is increased lactate production. HIF- $1 \alpha$ facilitates this metabolic switch by binding to hypoxia response elements in target genes [30,31], such as the glucose transporter GLUT1 [32] and glycolytic enzymes. HIF-1 $\alpha$ induces expression of lactate dehydrogenase (LDH) [33], which catalyzes lactate production from pyruvate, thereby limiting the production of acetyl-CoA for the TCA cycle. HIF-1 $\alpha$ also increases the expression of pyruvate dehydrogenase kinase [34, 35], which inhibits pyruvate dehydrogenase, an enzyme that catalyzes the formation of acetyl-CoA from pyruvate. HIF-1 $\alpha$ promotes the glycolytic switch in hypoxic macrophages, and ATP levels are greatly reduced in HIF- $1 \alpha$-deficient macrophages [36].

A similar process occurs in LPS-activated macrophages under normoxic conditions [12, 37]. Furthermore, macrophage activation by LPS is dependent on glycolysis, as inhibition of glycolysis with 2-deoxyglucose (2DG) decreases the inflammatory response, whereas inhibitors of mitochondrial respiration have no such effect, consistent with the fact that oxidative metabolism is already shut down under such conditions [38]. 2DG decreases LPS-induced production of the inflammatory cytokine interleukin (IL)-1 $\beta$ and inhibits HIF-1 $\alpha$ activation [12]. HIF-1 $\alpha$-knockout macrophages also have decreased expression of iNOS after IFN $\gamma$ stimulation [39]. Bacterial infection induces HIF-1 $\alpha$ expression in macrophages, and HIF-1 $\alpha$-null macrophages are less well able to kill bacteria [40].

A key mechanism for HIF-1 $\alpha$ activation by LPS involves the mammalian target of rapamycin (mTOR). This serine/threonine protein kinase is active when nutrients are in abundance, and so is highly active in proliferating cells and metabolically demanding situations, e.g., after TLR stimulation [41]. mTOR helps cells meet this high metabolic demand by increasing HIF$1 \alpha$ expression, which in turn increases the expression of glycolytic genes, as well as inflammatory genes. mTOR mediates the increase in HIF- $1 \alpha$ expression by promoting translation of mRNAs containing 5'-terminal oligopyrimidine (5'-TOP) signals, motifs that are present in HIF-1 $\alpha$ mRNA [42]. Furthermore, the mTOR complex component Raptor interacts with HIF-1 $\alpha$ via an mTOR signaling motif located in HIF- $1 \alpha$, thereby promoting HIF$1 \alpha$ activity [43]. The LPS-induced increase in HIF-1 $\alpha$ expression level and activity therefore provides a further parallel with tumour cell metabolism. The mTOR-HIF-1 $\alpha$ axis also has a role in the memory function of the innate immune system, known as trained immunity. This memory component is based on epigenetic reprogramming of innate immune cells, as demonstrated by the epigenetic changes observed during the monocyte-to-macrophage differentiation induced by $\beta$-glucan training [44]. Trained monocytes rely on aerobic glycolysis, a metabolic programme orchestrated by an Akt-mTOR-HIF-1 $\alpha$ pathway. Mice with myeloid cell-specific HIF- $1 \alpha$ deficiency lack the ability to induce a trained innate immune response to sepsis [45].

Inhibition of glycolysis also blocks DC maturation in response to LPS, as evidenced by reduced expression of the costimulatory molecules CD80 and CD86 in the presence of 2DG [46]. Similarly to macrophages, HIF$1 \alpha$ is a key factor in the metabolic switch and immune function in DCs. Compared with hypoxic treatment, a classical inducer of HIF- $1 \alpha$, LPS stimulation in normoxia induces a greater amount of HIF-1 $\alpha$ protein and mRNA in DCs [46]. This is accompanied by increased induction of the HIF-1 $\alpha$ target genes GLUT1 and phosphoglycerate kinase (PGK). HIF-1 $\alpha$ deficiency in LPS-stimulated DCs reduces the levels of the costimulatory molecules CD80 and CD86 on the cell surface [46, 47], and these DCs are less well able to drive T-cell proliferation [46, 47]. DCs activated by TLR2 ligation also have increased 
levels of HIF-1 $\alpha$ [48]. This metabolic shift is evident in DCs in vivo, in response to poly(I:C)-stimulated type I IFN signaling [11]. DCs activated in this manner exhibit increased glycolysis and reduced mitochondrial respiration, as well as increased Hifla transcription. This response was impaired in mice lacking IFNAR, the type I IFN receptor [11]. In contrast to macrophages [12], however, HIF-1 $\alpha$ may not have a role in cytokine release from LPS-activated DCs [47].

LPS also promotes mTOR activation in murine DCs [49], though this may not translate to human DCs [50]. Rapamycin, an inhibitor of mTOR, abolishes LPS-induced IL-10 induction in murine DCs, which leads to increased IL-12 levels, as IL-10 inhibits the production of IL-12 in an autocrine manner [49]. DCs lacking tuberous sclerosis complex 1 (TSC1), an upstream negative regulator of mTOR, exhibit increased glycolysis and expression of glycolytic enzymes, including hexokinase-2 (HK2) and LDHA [51]. Surprisingly, DCs lacking TSC1 have increased mitochondrial respiration (as well as increased glycolysis), and are developmentally impaired [51]. Furthermore, TSC1-deficient DCs are less capable of inducing $T_{h} 1$ responses [51]. In another study, mTOR inhibition in DCs promotes their long-term survival and their ability to stimulate T-cell responses [50]. DCs activated in the presence of rapamycin are better able to induce the primary expansion of $\mathrm{CD} 8^{+} \mathrm{T}$ cells in response to LPS plus OVA antigen, as well as the later establishment of a memory $\mathrm{CD} 8^{+} \mathrm{T}$-cell population, compared to DCs without rapamycin treatment [50]. The downstream effects of mTOR inhibition in DCs may depend on the particular population of DCs under investigation, as well as the experimental context, and further work is needed to clarify the role of mTOR in DC function.

HIF- $1 \alpha$ and mTOR are central regulators of the metabolic switch in both macrophages and DCs, with a wide range of downstream targets that facilitate this process. The mTOR-HIF-1 $\alpha$ pathway is complex, and more work is needed to dissect the effects of mTOR-HIF-1 $\alpha$ signaling in various inflammatory situations.

\section{$u$-PFK2 and glycolysis in macrophages}

Another LPS-regulated target involved in the switch to glycolysis is the $p f k f b 3$ gene encoding u-PFK2 (PFKFB3), an isoform of 6-phosphofructo-2-kinase/ fructose-2,6-bisphosphatase (PFK-2) [52, 53]. PFK-2 regulates intracellular levels of the glycolytic intermediate fructose-2,6-bisphosphate (F-2,6-BP), as it has dual kinase:bisphosphatase activities and thus catalyzes both the synthesis and degradation of this intermediate. F-2,6BP allosterically activates 6-phosphofructo-1-kinase, thereby increasing flux through the glycolytic pathway
[54]. u-PFK2 is the isoform of this enzyme that has the highest kinase:bisphosphatase activity ratio, and therefore maintains the highest levels of the biphosphorylated form of the metabolite, F-2,6-BP [55, 56]. Interestingly, $p f k f b 3$ is a HIF-1 $\alpha$ target gene in response to hypoxia in human glioblastoma cell lines and mouse embryonic fibroblasts [57], and thereby provides another mechanism by which HIF-1 $\alpha$ promotes glycolysis.

The purine nucleoside adenosine, a metabolite of ATP, can synergize with LPS to boost glycolysis in macrophages [58]. LPS stimulation of macrophages increases the expression of the $A_{2 A}$ and $A_{2 B}$ cell surface receptors $\left(A_{2 A} R\right.$ and $\left.A_{2 B} R\right)$ for adenosine [58]. Adenosine binds to these receptors, which further increases LPS-induced expression of $p f k f b 3$. This synergistic induction of $p f k f b 3$ is not mediated by HIF-1 $\alpha$, but by the transcription factor Sp1 [58]. Adenosine also synergizes with LPS to boost lactate production, indicating that adenosine enhances the LPS-induced increase in glycolysis [58]. In general, however, adenosine signaling often acts in an anti-inflammatory manner. For example, following LPS stimulation, $\mathrm{A}_{2 \mathrm{~B}} \mathrm{R}$-deficient macrophages showed increased production of $\mathrm{NO}$ and pro-inflammatory cytokines [59]. Adenosine boosts $E$. coli-induced production of the anti-inflammatory cytokine IL-10 in peritoneal macrophages, and $E$. coli is unable to induce IL-10 production in macrophages lacking $\mathrm{A}_{2 \mathrm{~A}} \mathrm{R}$ [60]. Interestingly, inosine, a breakdown product of adenosine, exerts anti-inflammatory effects by decreasing the LPS-induced production of TNF- $\alpha$ and IL-1 in mouse peritoneal macrophages [61].

Taken together, increasing u-PFK2 expression level is a critical step in the induction of glycolysis in activated innate immune cells.

\section{AMPK and activation of macrophages and DCs}

Finally, LPS also decreases activation of the energy-sensing enzyme AMP-activated protein kinase (AMPK) in macrophages [62]. AMPK activity opposes the metabolic switch towards glycolysis in DCs [10]. AMPK is active when the cellular energy charge is low, as binding of either AMP or ADP to this kinase renders it more susceptible to stimulatory phosphorylation by its upstream kinases, liver kinase B1 (LKB1) and calmodulin-dependent protein kinase kinase 2 (CaMKK2). As the function of AMPK is to conserve energy when it is limited, this enzyme inhibits anabolic pathways, such as gluconeogenesis, and drives catabolic pathways, including $\beta$-oxidation of fatty acids. AMPK induces the expression of proteins involved in OXPHOS, such as peroxisome proliferator-activated receptor (PPAR)- $\gamma$ coactivator (PGC) $1 \beta$, a transcriptional coactivator that promotes mitochondrial biogenesis [63], and the activities of mito- 
chondrial enzymes, including succinate dehydrogenase (SDH) [64]. AMPK also upregulates enzymes involved in fatty acid metabolism [64], such as carnitine palmitoyltransferase $1 \alpha(\mathrm{CPT} 1 \alpha)$ that promotes fatty acid uptake by mitochondria for $\beta$-oxidation. LPS-induced inhibition of AMPK dampens the activity of these pathways, which is useful to highly activated cells as they need to boost biosynthetic pathways to produce inflammatory mediators.

Consistent with its role in decreasing anabolism, AMPK can inhibit mTOR in various cell types to decrease protein synthesis, a downstream effect of mTOR $[65,66]$. Inhibition of AMPK by LPS may therefore represent another means by which LPS promotes mTOR activity and consequently HIF- $1 \alpha$ signaling, which could be another mechanism by which LPS induces the metabolic switch in innate immune cells.

Genetic manipulation of AMPK provides evidence for its role in inflammatory signaling. Macrophages and DCs derived from AMPK $\alpha 1^{-/-}$mice show increased production of TNF- $\alpha$ and IL- 6 , and decreased production of IL-10 in response to LPS [67]. Macrophages from mice lacking the AMPK $\beta 1$ subunit have reduced rates of palmitate oxidation, and as a consequence show increased production of the pro-inflammatory cytokines IL-1 $\beta$ and TNF- $\alpha$ [68]. LPS activation of DCs decreases stimulatory phosphorylation of AMPK, and shRNA targeting of AMPK results in increased IL-12p40 and CD86 expression [10].

AMPK can also modulate inflammatory signaling downstream of LPS by affecting transcription factors involved in the inflammatory response. AMPK antagonizes nuclear factor $\kappa \mathrm{B}(\mathrm{NF} \kappa \mathrm{B})$ signaling, a pathway responsible for the transcription of pro-inflammatory cytokines such as IL-1 $\beta$ and TNF- $\alpha$. AMPK stabilizes inhibitor of $\kappa \mathrm{B} \alpha(\mathrm{I} \kappa \mathrm{B} \alpha)$ and $\mathrm{p} 53$, both of which inhibit NF $\mathrm{B}$ activity in various cell types [69]. Furthermore, AMPK can activate Sirtuin 1 (SIRT1), an NAD-dependent deacetylase that has been shown to deacetylate and thus inactivate p65, a subunit of NFkB, in HEK 293T cells expressing p65 [70].

Overall, AMPK counters many metabolic changes induced in pro-inflammatory macrophages and DCs, and its inhibition by LPS allows these changes to proceed.

\section{Citrate in macrophages and DCs}

A key functional outcome for the switch to glycolysis is increased ATP production and PPP activity for biosynthesis. However, it has also recently been found that another metabolic event occurs in response to LPS earlier in the time course of macrophage activation (be- fore NO accumulation limits OXPHOS), which involves citrate. Citrate is a TCA cycle intermediate formed from oxaloacetate and acetyl-CoA by citrate synthase in the mitochondria. LPS increases expression of the mitochondrial citrate carrier, solute carrier family 25 member 1 (Slc25a1), via NFkB in macrophages [71]. This carrier transports citrate out of the mitochondrion in exchange for malate, causing cytosolic citrate accumulation. When ATP is present, the enzyme ATP-citrate lyase (ACLY) in the cytosol converts citrate back to oxaloacetate and acetyl-CoA. Acetyl-CoA is a source of acetyl groups for acetylation of histones [72]. Interestingly, the transcription of genes encoding glycolytic enzymes $H K 2$, phosphofructokinase-1 (PFK1) and LDHA is regulated downstream of ACLY. Silencing of ACLY suppressed expression of these genes, thereby decreasing glucose consumption. This effect was rescued by the addition of acetate, which can provide the acetyl groups needed for histone acetylation on these genes. Citrate metabolism can therefore influence the induction of glycolysis [72]. The increase in acetyl-CoA levels after TLR stimulation has another function in activated macrophages and DCs. Acetyl-CoA has been shown to help activated macrophages meet their increased biosynthetic demands, particularly in lipid biosynthesis. For example, ACLY is needed for the production of the eicosanoid prostaglandin E2 $\left(\mathrm{PGE}_{2}\right)$ in response to LPS or TNF- $\alpha$ stimulation in phorbol-12-myristate-13-acetate (PMA)-differentiated U937 cells, human monocytic cells from histiocytoma [73]. The function of acetyl-CoA in promoting lipid biosynthesis depends on acetyl-CoA carboxylase (ACC), which converts acetyl-CoA to malonyl-CoA, a metabolite that is used for fatty acid synthesis. In LPS-activated DCs, citrate is preferentially removed from the TCA cycle and used for de novo synthesis of fatty acids, and increased glycolytic flux is needed to support this metabolic shift [74]. This increase in glycolysis is mediated by the kinases TANK-binding kinase 1 (TBK1), inhibitor of $\kappa \mathrm{B}$ kinase $\varepsilon(\mathrm{IKK} \varepsilon)$ and Akt, which drive the association of the glycolytic enzyme $H K 2$ with the mitochondria. Genetic silencing of Slc25a1 or inhibition of fatty acid synthesis by blocking the activity of ACC both result in the impairment of LPS-induced DC activation. The decreased fatty acid biosynthesis limits membrane production needed for the expansion of endoplasmic reticulum and Golgi, which then decreases protein production, including the generation of cytokines needed for T-cell activation by DCs [74]. Consistent with this, liver DCs with a high lipid content are immunogenic, inducing T-cell and NK-cell activation, while those with lower lipid concentrations are more tolerogenic and induce regulatory T cells [75]. 
ACLY-catalyzed citrate metabolism also contributes to the production of inflammatory mediators such as NO and ROS. Oxaloacetate, the other product of citrate metabolism, is further metabolized to pyruvate with the concomitant generation of NADPH from $\mathrm{NADP}^{+}$. NADPH and molecular oxygen can be used by NADPH oxidase to generate ROS. NADPH is also required as a substrate for the conversion of L-arginine to $\mathrm{NO}$ and citrulline. NO could then participate in the LPS-induced metabolic switch by inhibiting mitochondrial OXPHOS, as could ROS, as they stabilize HIF-1 $\alpha$ [76]. Activation of macrophages by either LPS, TNF- $\alpha$, or IFN $\gamma$ alone, or a combination of TNF- $\alpha$ and IFN $\gamma$ induces ACLY expression, and inhibition of ACLY activity decreases the production of NO and ROS [73]. Inhibition of Slc25a1 or its silencing also results in decreased production of NO, ROS, and $\mathrm{PGE}_{2}$ in U937 cells [71].

These changes in citrate transport and metabolism (Figure 3) would therefore appear to be critical for the activation of macrophages and DCs by LPS, the latter in turn triggers T-cell activation and adaptive immunity.

\section{Succinate as a signal in macrophages and DCs}

Succinate, another TCA cycle intermediate, has been found to be involved in macrophage activation by LPS [12]. Succinate levels are elevated in macrophages after LPS stimulation [12], and are also increased in mouse models of obesity and diabetes [77], metabolic disorders associated with inflammation. This LPS-stimulated increase in succinate levels does not originate from the TCA cycle, as the activity of this system is dampened in response to LPS [10, 12, 25]. Glutamine metabolism provides the source for this LPS-induced increase in succinate generation, via anaplerosis $[52,78]$ proceeding through $\alpha$-ketoglutarate $(\alpha-K G)$, as well as via the $\gamma$-aminobutyric acid (GABA) shunt [12]. Succinate can also be generated via the glyoxylate shunt, a system that operates in bacteria, fungi, protists, and plants [79-81]. The first enzyme of this shunt, isocitrate lyase (ICL), converts isocitrate to succinate and glyoxylate. ICL has been shown to have a positive role in the persistence of M. tuberculosis in LPS-activated macrophages [82]. However, it is unclear whether LPS increases succinate production via the ICL pathway in mammals upon microbial infection. Interestingly, mammalian macrophages highly express immunoresponsive gene 1 (Irg1) during inflammation, which catalyzes the generation of itaconic acid from the TCA cycle intermediate cis-aconitate [83]. Itaconic acid is a metabolite thought to inhibit ICL and thereby decrease microbial growth $[83,84]$. The role of itaconic acid in the metabolism of innate immune cells warrants further studies.

Succinate stabilizes HIF- $1 \alpha$, thereby promoting the switch to glycolysis and driving inflammation. It accomplishes this by inhibiting the activity of prolyl hydroxylase (PHD) enzymes, so that they can no longer hydroxylate and destabilize HIF- $1 \alpha$. HIF- $1 \alpha$ can then interact with coactivators to induce the glycolytic metabolic program, and drive inflammation by increasing transcription of IL-1 $\beta$ [12]. Vigabatrin, an inhibitor of the key GABA shunt enzyme GABA transaminase, is protective in a mouse model of LPS-induced sepsis [12].

Extracellular succinate can signal via the G protein-coupled receptor (GPR) 91. GPR91 is highly expressed by DCs, and can synergize with TLR signaling to boost the induction of the pro-inflammatory cytokine TNF- $\alpha$ by the TLR3 ligand poly(I:C) or the TLR7 ligand imiquimod [85].

Succinate can also act as a substrate for succinylation, a post-translational modification of proteins in which a succinyl group is added to a lysine residue. LPS increases succinylation of multiple proteins [12]. Succinylated targets include the glycolytic enzyme glyceraldehyde 3-phosphate dehydrogenase (GAPDH), as well as malate dehydrogenase $(\mathrm{MDH}), \mathrm{LDH}$, and glutamate carrier 1 [12]. It is not yet known whether and how succinylation affects the activity of these proteins.

Succinate accumulates during ischaemia in vivo in a mouse model of ischaemia-reperfusion (IR), suggesting that it may have a pathological role in IR. Succinate drives the production of mitochondrial ROS after reperfusion, as demonstrated in a primary cardiomyocyte model of IR injury. Decreasing succinate levels reduces IR injury in mouse models of stroke and heart attack [86]. The effect of succinate on mitochondrial ROS production may also occur in innate immune cells, representing another possible mechanism underlying the metabolic switch in these cells.

Clearly, succinate is an important signaling molecule in innate immune cells, and may also represent a therapeutic target in a variety of pathologies.

\section{M2 macrophages have a different metabolic profile compared to M1 macrophages}

All of the above events have been shown to be critical in macrophages and DCs activated by classical stimuli such as LPS, IFN $\gamma$ and bacteria including E. coli. Such classically activated macrophages have been termed "M1 macrophages" and are positively associated with inflammation. Intriguingly, the so-called M2 or alternatively activated macrophages have a different metabolic profile and inflammatory phenotype $[87,88]$. These two 


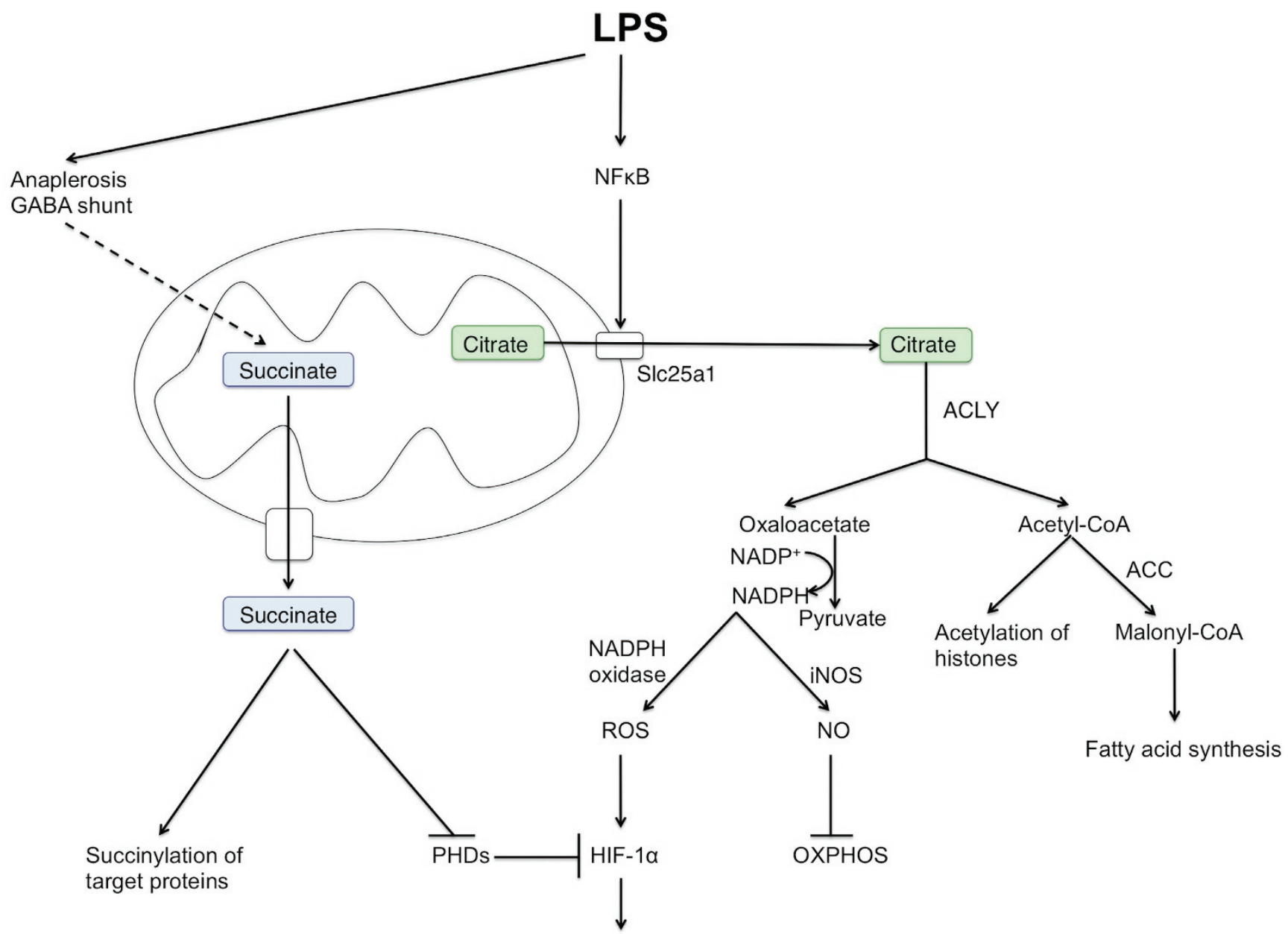

\section{Inflammation}

Figure 3 Roles of citrate and succinate in macrophage and DC activation by LPS. Metabolic intermediates of the TCA cycle, such as citrate and succinate, can act as signaling molecules in macrophages and DCs, even when TCA cycle activity is decreased. LPS increases expression of the citrate carrier, Slc25a1, which could lead to increased transport of citrate out of the mitochondria. Citrate is then metabolized to acetyl-CoA and oxaloacetate by ACLY. Acetyl-CoA is used in fatty acid synthesis and also provides acetyl groups for acetylation of histone proteins. The conversion of oxaloacetate to pyruvate generates NADPH, which serves as a substrate in both iNOS-catalyzed NO production and NADPH oxidase-catalyzed ROS generation. NO nitrosylates and inhibits components of the mitochondrial electron transport chain and thus inhibits OXPHOS. ROS can

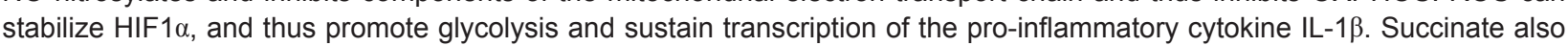
promotes HIF-1 $\alpha$ stabilization by inhibiting PHD enzymes, which, when active, hydroxylate and increase the degradation of $\mathrm{HIF}-1 \alpha$. Succinate is also used to succinylate proteins, a post-translational modification with as yet unknown consequences. Sources of succinate in an LPS-activated macrophage include glutamine metabolism (anaplerosis and the GABA shunt), and possibly the glyoxylate shunt.

phenotypes are not rigid and there may in fact exist a "spectrum" of macrophage activation, involving macrophages with different metabolic and inflammatory signatures, and with different roles in host defense against various pathogens, as well as in wound healing and the resolution of inflammation [89, 90]. M2 macrophages exhibit increased flux through OXPHOS and higher expression of anti-inflammatory cytokines such as IL-10, but have decreased production of NO and TNF- $\alpha$ [88]. They are involved in immune responses to parasites [91], as evidenced by their role in the induction of $\mathrm{T}_{\mathrm{h}} 2$-type responses in disease models of parasitic infection [92]. Why glycolysis is needed for M1 macrophage activation, whilst OXPHOS promotes the M2 macrophage phenotype, is not known. The cytokines IL-4 and IL-13 are key inducers of M2 macrophage polarization. 
Many of the processes that drive the glycolytic switch in M1 macrophages are downregulated in M2 macrophages (Figure 4). First, arginase 1 (Arg-1) is highly expressed in M2 macrophages, while iNOS expression is decreased [93]. M1 macrophages have increased levels of citrulline and the inflammatory mediator $\mathrm{NO}$, while in M2 macrophages, arginine is preferentially metabolized to urea and ornithine [94]. Ornithine can be further metabolized to polyamines and proline. Schistosome eggs induce Arg-1 expression in infected mice, and the combination of IL-4 and IL-13 increases proline production by macrophages [93]. This increased proline production could contribute to granuloma formation and liver fibrosis in infected mice, as both of these are enhanced when proline synthesis is boosted [93]. However, there is also conflicting evidence suggesting that Arg-1-expressing macrophages suppress schistosome-induced fibrosis during chronic infection [95]. Interestingly, Arg-1 can be induced in classically activated macrophages by intracellular infection with mycobacteria, and functions in this context to suppress NO production [96]. M. tuberculosis-infected mice lacking Arg-1 expression in macrophages are better able to clear the bacteria [96].
Secondly, IL-4 and IL-13 induce oxidative metabolism by inhibiting mTOR via activation of its upstream negative regulators TSC1 and TSC2. Macrophages lacking these negative regulators cannot switch towards OXPHOS, and Arg-1 expression is reduced [41]. Inhibition of mTOR by rapamycin treatment or transient TSC2 expression [43] can also lead to a decrease in HIF-1 $\alpha$ levels, and therefore could result in reduced HIF-1 $\alpha$-dependent glycolytic and inflammatory gene expression [97]. In contrast to M1 macrophages, expression of the HIF- $2 \alpha$ isoform of HIF is induced in IL-4and IL-13-stimulated macrophages, while its levels are reduced in macrophages treated with LPS and IFN $\gamma$ [39]. HIF- $2 \alpha$-knockout macrophages have decreased levels of Arg-1, while iNOS is unaffected [39]. Interestingly, HIF$2 \alpha$ may still have some role in M1-like macrophages, particularly those in hypoxic conditions [98]. IL-1 $\beta$, IL12 , and TNF- $\alpha$ levels are decreased in LPS-stimulated mice with macrophages lacking HIF-2 $\alpha$, while IL-10 levels are increased. However, HIF-2 $\alpha$ may not have a role in induction of glycolysis and maintenance of ATP levels [98].

Thirdly, M2 macrophages predominantly express
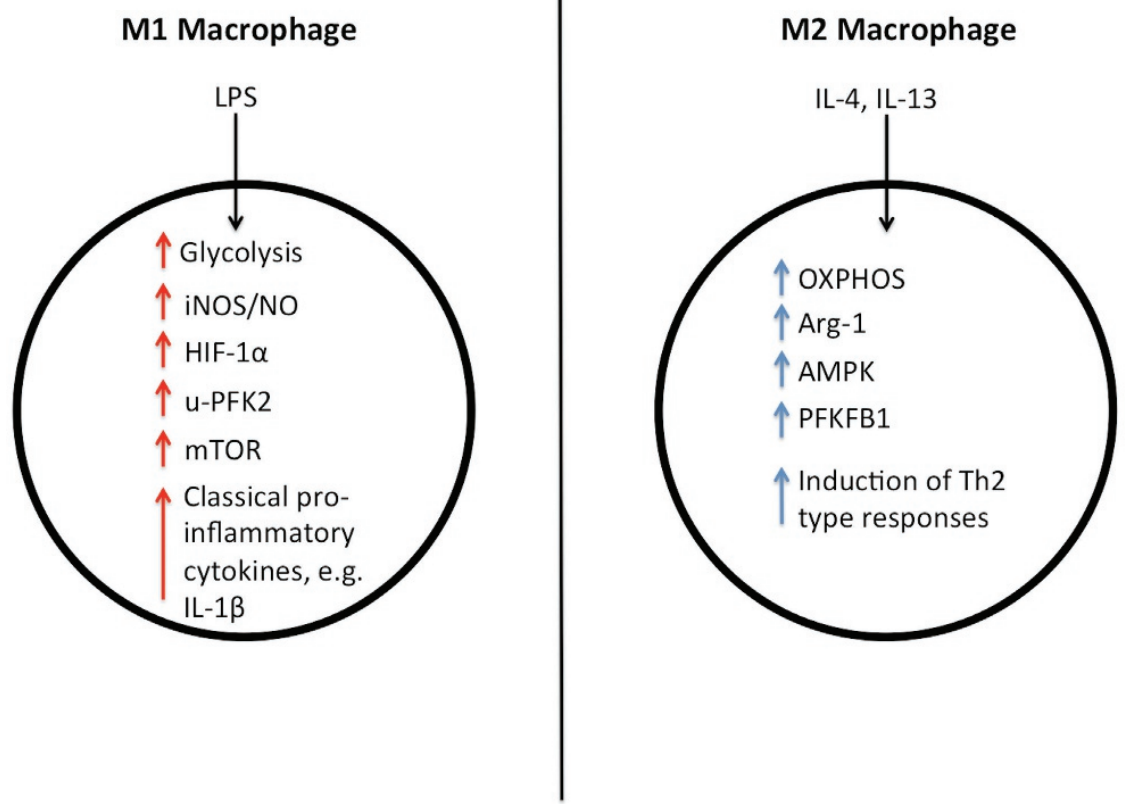

Figure 4 Metabolic differences between M1 and M2 macrophages. M1 macrophages rely on glycolysis for ATP production and have increased levels of iNOS, HIF-1 $\alpha$ and u-PFK2, while M2 macrophages are fueled by OXPHOS and have increased levels of Arg-1, AMPK and PFKFB1. M1 macrophages release pro-inflammatory cytokines such as IL-1 $1 \beta$, while M2 macrophages are involved in the response to parasite infection, as well as in wound healing, and they release the anti-inflammatory cytokine IL-10. In fact, it is thought that a spectrum of macrophage activation exists, with different populations of macrophages exhibiting different inflammatory and metabolic phenotypes. 
PFKFB1, an isoform of PFK2 [52]. PFKFB1 has higher bisphosphatase activity than u-PFK2 $[55,56]$, and therefore can more readily break down F-2,6-BP into fructose-6-phosphate, decreasing glycolytic activity.

Furthermore, AMPK activity is high in M2 macrophages, and drives production of the anti-inflammatory cytokine IL-10 [62]. M2 macrophages are fueled by fatty acid uptake and oxidation in the mitochondria, a metabolic program controlled by AMPK and its downstream targets, which include PPARs. IL-4 and IL-13 increase the expression of PPARs and their coactivator protein PGC1 $\beta$, leading to increased expression of proteins of the mitochondrial respiratory chain. Knockdown of PGC1 $\beta$ antagonizes this anti-inflammatory skewing of macrophage metabolism [63]. IL-4 also enhances PPAR $\gamma$ activity via signal transducer and activator of transcription 6 (STAT6) in macrophages and DCs [99]. PPAR $\gamma$ boosts the ability of IL-4 to induce arginase expression in macrophages, and also has a role in the IL-4-stimulated increase in fatty acid $\beta$-oxidation in these macrophages [100]. AMPK also inhibits mTOR activity by activating TSC2 [65], as well as by directly phosphorylating the mTOR complex subunit Raptor [66]. Pharmacological activation of AMPK supports the view that it has anti-inflammatory activity. Treatment with the specific AMPK activator A769662 increases fatty acid oxidation and decreases activation of JNK, which is involved in inflammatory signaling [68]. In addition metformin, an AMPK activator and anti-diabetic drug, decreases inflammation in experimental autoimmune encephalomyelitis (EAE), a mouse model of multiple sclerosis [101].

There are other factors that contribute to a more M2like phenotype in macrophages. Peritoneal macrophages lacking the transcription factor Gata6 are more like alternatively activated macrophages, expressing high levels of Arg-1 [102]. Gata6 is also involved in acetyl-CoA metabolism. Gata6-deficient macrophages have drastically reduced expression of aspartoacylase (AspA), an enzyme involved in acetyl-CoA synthesis. The decreased AspA expression does not seem to affect macrophage polarization, but may render peritoneal macrophages more susceptible to death.

Another intriguing enzyme recently implicated in macrophage polarization is the carbohydrate kinase-like protein (CARKL), a sedoheptulose kinase that catalyzes the production of sedoheptulose-7-phosphate. Its activity limits the flux into PPP, thereby decreasing levels of NADPH and glutathione [14]. Knockdown of CARKL potentiates LPS-induced cytokine production and M1 macrophage polarization, while CARKL overexpression decreases IL-6 secretion in response to LPS. Consistent with this, LPS treatment decreases CARKL levels, while M2 macrophage polarization conditions increase CARKL levels [14].

Metabolic differences therefore appear to lie at the heart of the differing functions of M1 and M2 macrophages.

\section{Final remarks}

Despite early observations about the metabolism of innate immune cells, it has taken some time to begin to mechanistically explain these observations and appreciate their impact on immune function. Moreover, there is vast scope for further discovery. There are many metabolites that are much less well characterized than those described here, and new ones are constantly being discovered, such as itaconic acid, discussed above [83].

Furthermore, insight into the interface between metabolism and innate immunity could provide novel tools to manipulate either of these cellular activities. This could be particularly relevant in the context of disease. For example, inflammation has a role in promoting insulin resistance and pancreatic $\beta$-cell dysfunction $[103,104]$. The pro-inflammatory cytokine IL- $1 \beta$ induces apoptosis of $\beta$-cells [103] and therefore participates in the pathogenesis of type 2 diabetes mellitus [105, 106]. Conversely, metabolic pathways influence the course of immune diseases, as illustrated by the fact that the metabolic drugs metformin and AICAR, both of which can activate AMPK, can ameliorate inflammation [101, 107]. Metabolite screening could also become important in clinical diagnosis, as metabolic intermediates are often present at abnormal levels in diseased states [108].

Finally, improvements in metabolomics and other metabolism-based technologies should aid greatly in advancing the current knowledge of immunometabolism. There are wide areas of this field still to be explored, including epigenetic regulation of immune cell metabolism and inflammation [45], post-translational modifications utilizing metabolites [109-111], and the discovery of as yet unknown immunometabolic signaling pathways.

\section{References}

1 Janeway CA Jr, Medzhitov R. Innate immune recognition. Annu Rev Immunol 2002; 20:197-216.

2 Chen GY, Nunez G. Sterile inflammation: sensing and reacting to damage. Nat Rev Immunol 2010; 10:826-837.

3 Warburg O, Wind F, Negelein E. The metabolism of tumors in the body. J Gen Physiol 1927; 8:519-530.

4 Sbarra AJ, Karnovsky ML. The biochemical basis of phagocytosis. I. Metabolic changes during the ingestion of particles by polymorphonuclear leukocytes. J Biol Chem 1959; 234:1355-1362. 
5 Guthrie LA, McPhail LC, Henson PM, Johnston Jr RB. Priming of neutrophils for enhanced release of oxygen metabolites by bacterial lipopolysaccharide. Evidence for increased activity of the superoxide-producing enzyme. J Exp Med 1984; 160:1656-1671.

6 Borregaard N, Herlin T. Energy metabolism of human neutrophils during phagocytosis. J Clin Invest 1982; 70:550-557.

7 Hard GC. Some biochemical aspects of the immune macrophage. Br J Exp Pathol 1970; 51:97-105.

8 Newsholme P, Gordon S, Newsholme EA. Rates of utilization and fates of glucose, glutamine, pyruvate, fatty acids and ketone bodies by mouse macrophages. Biochem $J 1987$; 242:631-636.

9 Newsholme P, Curi R, Gordon S, Newsholme EA. Metabolism of glucose, glutamine, long-chain fatty acids and ketone bodies by murine macrophages. Biochem $J$ 1986; 239:121125.

10 Krawczyk CM, Holowka T, Sun J, et al. Toll-like receptor-induced changes in glycolytic metabolism regulate dendritic cell activation. Blood 2010; 115:4742-4749.

11 Pantel A, Teixeira A, Haddad E, Wood EG, Steinman RM, Longhi MP. Direct type I IFN but not MDA5/TLR3 activation of dendritic cells is required for maturation and metabolic shift to glycolysis after poly IC stimulation. PLoS Biol 2014; 12:e1001759.

12 Tannahill GM, Curtis AM, Adamik J, et al. Succinate is an inflammatory signal that induces IL-1beta through HIF-1alpha. Nature 2013; 496:238-242.

13 Freemerman AJ, Johnson AR, Sacks GN, et al. Metabolic reprogramming of macrophages: glucose transporter 1 (GLUT1)-mediated glucose metabolism drives a proinflammatory phenotype. J Biol Chem 2014; 289:7884-7896.

14 Haschemi A, Kosma P, Gille L, et al. The sedoheptulose kinase CARKL directs macrophage polarization through control of glucose metabolism. Cell Metab 2012; 15:813-826.

15 Bedard K, Krause KH. The NOX family of ROS-generating NADPH oxidases: physiology and pathophysiology. Physiol Rev 2007; 87:245-313.

16 Vazquez A, Liu J, Zhou Y, Oltvai ZN. Catabolic efficiency of aerobic glycolysis: the Warburg effect revisited. BMC Syst Biol 2010; 4:58.

17 West AP, Brodsky IE, Rahner C. TLR signalling augments macrophage bactericidal activity through mitochondrial ROS. Nature 2011; 472:476-480.

18 Palsson-McDermott EM, O'Neill LA. The Warburg effect then and now: from cancer to inflammatory diseases. Bioessays 2013; 35:965-973.

19 Qualls JE, Subramanian C, Rafi W, et al. Sustained generation of nitric oxide and control of mycobacterial infection requires argininosuccinate synthase 1. Cell Host Microbe 2012; 12:313-323.

20 Lorsbach RB, Murphy WJ, Lowenstein CJ, Snyder SH, Russell SW. Expression of the nitric oxide synthase gene in mouse macrophages activated for tumour cell killing. $J$ Biol Chem 1993; 268:1908-1913.

$21 \mathrm{Lu} \mathrm{L}$, Bonham CA, Chambers FG, et al. Induction of nitric oxide synthase in mouse dendritic cells by IFN-gamma, endotoxin, and interaction with allogeneic T cells: nitric oxide production is associated with dendritic cell apoptosis. J Immunol
1996; 157:3577-3586.

22 Drapier JC, Hibbs Jr JB. Differentiation of murine macrophages to express nonspecific cytotoxicity for tumor cells results in L-arginine-dependent inhibition of mitochondrial iron-sulfur enzymes in the macrophage effector cells. J Immunol 1988; 140:2829-2838.

23 Clementi E, Brown GC, Feelisch M, Moncada S. Persistent inhibition of cell respiration by nitric oxide: crucial role of S-nitrosylation of mitochondrial complex I and protective action of glutathione. Proc Natl Acad Sci USA 1998; 95:76317636.

24 Cleeter MW, Cooper JM, Darley-Usmar VM, Moncada S, Schapira AH. Reversible inhibition of cytochrome c oxidase, the terminal enzyme of the mitochondrial respiratory chain, by nitric oxide. Implications for neurodegenerative diseases. FEBS Lett 1994; 345:50-54.

25 Everts B, Amiel E, van der Windt GJ, et al. Commitment to glycolysis sustains survival of NO-producing inflammatory dendritic cells. Blood 2012; 120:1422-1431.

26 Lou Y, Zhang G, Geng M, Zhang W, Cui J, Liu S. TIPE2 negatively regulates inflammation by switching arginine metabolism from nitric oxide synthase to arginase. PLoS One 2014; 9:e96508.

27 Gobert AP, McGee DJ, Akhtar M, et al. Helicobacter pylori arginase inhibits nitric oxide production by eukaryotic cells: a strategy for bacterial survival. Proc Natl Acad Sci USA 2001; 98:13844-13849.

28 Doulias PT, Tenopoulou M, Greene JL, Raju K, Ischiropoulos $\mathrm{H}$. Nitric oxide regulates mitochondrial fatty acid metabolism through reversible protein S-nitrosylation. Sci Signal 2013; 6:rs1.

29 Denko NC. Hypoxia, HIF1 and glucose metabolism in the solid tumour. Nat Rev Cancer 2008; 8:705-713.

30 Semenza GL, Nejfelt MK, Chi SM, Antonarakis SE. Hypoxia-inducible nuclear factors bind to an enhancer element located 3' to the human erythropoietin gene. Proc Natl Acad Sci USA 1991; 88:5680-5684.

31 Mole DR, Blancher C, Copley RR, et al. Genome-wide association of hypoxia-inducible factor (HIF)-1alpha and HIF-2alpha DNA binding with expression profiling of hypoxia-inducible transcripts. $J$ Biol Chem 2009; 284:16767-16775.

32 Chen C, Pore N, Behrooz A, Ismail-Beigi F, Maity A. Regulation of glut 1 mRNA by hypoxia-inducible factor-1. Interaction between H-ras and hypoxia. J Biol Chem 2001; 276:95199525.

33 Semenza, GL, Jiang BH, Leung SW, et al. Hypoxia response elements in the aldolase A, enolase 1, and lactate dehydrogenase A gene promoters contain essential binding sites for hypoxia-inducible factor 1. J Biol Chem 1996; 271:3252932537.

34 Kim JW, Tchernyshyov I, Semenza GL, Dang CV. HIF-1-mediated expression of pyruvate dehydrogenase kinase: a metabolic switch required for cellular adaptation to hypoxia. Cell Metab 2006; 3:177-185.

35 Papandreou I, Cairns RA, Fontana L, Lim AL, Denko NC. HIF-1 mediates adaptation to hypoxia by actively downregulating mitochondrial oxygen consumption. Cell Metab 2006; 3:187-197.

36 Cramer T, Yamanishi Y, Clausen BE, et al. HIF-1alpha is 
essential for myeloid cell-mediated inflammation. Cell 2003; 112:645-657.

37 Blouin CC, Pagé EL, Soucy GM, Richard DE. Hypoxic gene activation by lipopolysaccharide in macrophages: implication of hypoxia-inducible factor 1alpha. Blood 2004; 103:11241130.

38 Kellett DN. 2-Deoxyglucos and inflammation. J Pharm Pharmacol 1966; 18:199-200.

39 Takeda N, O'Dea EL, Doedens A, et al. Differential activation and antagonistic function of HIF- $\alpha$ isoforms in macrophages are essential for NO homeostasis. Genes Dev 2010; 24:491501.

40 Peyssonnaux C, Datta V, Cramer T, et al. HIF-1alpha expression regulates the bactericidal capacity of phagocytes. $J$ Clin Invest 2005; 115:1806-1815.

41 Byles V, Covarrubias AJ, Ben-Sahra I. The TSC-mTOR pathway regulates macrophage polarization. Nat Commun 2013; 4: 2834.

42 Huo Y, Iadevaia V, Yao Z, et al. Stable isotope-labelling analysis of the impact of inhibition of the mammalian target of rapamycin on protein synthesis. Biochem $J$ 2012; 444:141151.

43 Land SC, Tee AR. Hypoxia-inducible factor 1alpha is regulated by the mammalian target of rapamycin (mTOR) via an mTOR signaling motif. J Biol Chem 2007; 282:20534-20543.

44 Saeed S, Quintin J, Kerstens HH, et al. Epigenetic programming of monocyte-to-macrophage differentiation and trained innate immunity. Science 2014; 345:1251086.

45 Cheng SC, Quintin J, Cramer RA, et al. mTOR- and HIF-1alpha-mediated aerobic glycolysis as metabolic basis for trained immunity. Science 2014; 345:1250684.

46 Jantsch J, Chakravortty D, Turza N, et al. Hypoxia and hypoxia-inducible factor-1 alpha modulate lipopolysaccharide-induced dendritic cell activation and function. $J$ Immunol 2008; 180:4697-4705.

47 Bhandari T, Olson J, Johnson RS, Nizet V. HIF-1alpha influences myeloid cell antigen presentation and response to subcutaneous OVA vaccination. J Mol Med 2013; 91:1199-1205.

48 Spirig R, Djafarzadeh S, Regueira T, et al. Effects of TLR agonists on the hypoxia-regulated transcription factor HIF-1alpha and dendritic cell maturation under normoxic conditions. PLoS One 2010; 5:e010983.

49 Ohtani M, Nagai S, Kondo S, et al. Mammalian target of rapamycin and glycogen synthase kinase 3 differentially regulate lipopolysaccharide-induced interleukin-12 production in dendritic cells. Blood 2008; 112:635-643.

50 Amiel E, Everts B, Freitas TC, et al. Inhibition of mechanistic target of rapamycin promotes dendritic cell activation and enhances therapeutic autologous vaccination in mice. J Immunol 2012; 189:2151-2158.

51 Wang Y, Huang G, Zeng H, Yang K, Lamb RF, Chi H. Tuberous sclerosis 1 (Tsc1)-dependent metabolic checkpoint controls development of dendritic cells. Proc Natl Acad Sci USA 2013; 110:E4894-E4903.

52 Rodríguez-Prados JC, Través PG, Cuenca J, et al. Substrate fate in activated macrophages: a comparison between innate, classic, and alternative activation. J Immunol 2010; 185:605614.

53 Chesney J, Mitchell R, Benigni F, et al. An inducible gene product for 6-phosphofructo-2-kinase with an AU-rich instability element: role in tumor cell glycolysis and the Warburg effect. Proc Natl Acad Sci USA 1999; 96:3047-3052.

54 Pilkis SJ, El-Maghrabi MR, McGrane MM, Pilkis J, Claus TH. The role of fructose 2,6-bisphosphate in regulation of fructose-1,6-bisphosphatase. J Biol Chem 1981; 256:1148911495.

55 Sakata J, Abe Y, Uyeda K. Molecular cloning of the DNA and expression and characterization of rat testes fructose-6-phosphate,2-kinase:fructose-2,6-bisphosphatase. J Biol Chem 1991; 266:15764-15770.

56 Lee YH, Li Y, Uyeda K, Hasemann CA. Tissue-specific structure/function differentiation of the liver isoform of 6-phosphofructo-2-kinase/fructose-2,6-bisphosphatase. J Biol Chem 2003; 278:523-530.

57 Obach M, Navarro-Sabaté A, Caro J, et al. 6-Phosphofructo-2-kinase (pfkfb3) gene promoter contains hypoxia-inducible factor-1 binding sites necessary for transactivation in response to hypoxia. J Biol Chem 2004; 279:53562-53570.

58 Ruiz-Garcia A, Monsalve E, Novellasdemunt L, et al. Cooperation of adenosine with macrophage Toll-4 receptor agonists leads to increased glycolytic flux through the enhanced expression of PFKFB3 gene. J Biol Chem 2011; 286:1924719258.

59 Belikoff BG, Hatfield S, Georgiev P, et al. A2B adenosine receptor blockade enhances macrophage-mediated bacterial phagocytosis and improves polymicrobial sepsis survival in mice. J Immunol 2011; 186:2444-2453.

60 Csóka B, Németh ZH, Virág L, et al. A2A adenosine receptors and $\mathrm{C} / \mathrm{EBPb}$ ta are crucially required for IL-10 production by macrophages exposed to Escherichia coli. Blood 2007; 110:2685-2695.

61 Hasko G, Kuhel DG, Németh ZH, et al. Inosine inhibits inflammatory cytokine production by a posttranscriptional mechanism and protects against endotoxin-induced shock. $J$ Immunol 2000; 164:1013-1019.

62 Sag D, Carling D, Stout RD, Suttles J. Adenosine 5'-monophosphate-activated protein kinase promotes macrophage polarization to an anti-inflammatory functional phenotype. $J$ Immunol 2008; 181:8633-8641.

63 Vats D, Mukundan L, Odegaard JI, et al. Oxidative metabolism and PGC-1beta attenuate macrophage-mediated inflammation. Cell Metab 2006; 4:13-24.

64 Winder WW, Holmes BF. Activation of AMP-activated protein kinase increases mitochondrial enzymes in skeletal muscle. J Appl Physiol (1985) 2000; 88:2219-2226.

65 Inoki K, Zhu T, Guan KL. TSC2 mediates cellular energy response to control cell growth and survival. Cell 2003; 115:577-590.

66 Gwinn DM, Shackelford DB, Egan DF, et al. AMPK phosphorylation of raptor mediates a metabolic checkpoint. Mol Cell 2008; 30:214-226.

67 Carroll KC, Viollet B, Suttles J. AMPKalphal deficiency amplifies proinflammatory myeloid APC activity and CD40 signaling. J Leukoc Biol 2013; 94:1113-1121.

68 Galic S, Fullerton MD, Schertzer JD, et al. Hematopoietic AMPK beta 1 reduces mouse adipose tissue macrophage inflammation and insulin resistance in obesity. J Clin Invest 2011; 121:4903-4915. 
69 Ak P, Levine AJ. p53 and NF-kappaB: different strategies for responding to stress lead to a functional antagonism. FASEB $J$ 2010; 24:3643-3652.

70 Yeung F, Hoberg JE, Ramsey CS, et al. Modulation of NF-kappaB-dependent transcription and cell survival by the SIRT1 deacetylase. EMBO J 2004; 23:2369-2380.

71 Infantino V, Convertini P, Cucci L, et al. The mitochondrial citrate carrier: a new player in inflammation. Biochem J 2011; 438:433-436.

72 Wellen KE, Hatzivassiliou G, Sachdeva UM, Bui TV, Cross JR, Thompson CB. ATP-citrate lyase links cellular metabolism to histone acetylation. Science 2009; 324:1076-1080.

73 Infantino V, Iacobazzi V, Palmieri F, Menga A. ATP-citrate lyase is essential for macrophage inflammatory response. Biochem Biophys Res Commun 2013; 440:105-111.

74 Everts B, Amiel E, Huang SC, et al. TLR-driven early glycolytic reprogramming via the kinases TBK1-IKKvarepsilon supports the anabolic demands of dendritic cell activation. Nat Immunol 2014; 15:323-332.

75 Ibrahim J, Nguyen AH, Rehman A, et al. Dendritic cell populations with different concentrations of lipid regulate tolerance and immunity in mouse and human liver. Gastroenterology 2012; 143:1061-1072.

76 Calvani M, Comito G, Giannoni E, Chiarugi P. Time-dependent stabilization of hypoxia inducible factor- $1 \alpha$ by different intracellular sources of reactive oxygen species. PLoS One 2012; 7:e38388.

77 Sadagopan N, Li W, Roberds SL, et al. Circulating succinate is elevated in rodent models of hypertension and metabolic disease. Am J Hypertens 2007; 20:1209-1215.

78 Owen OE, Kalhan SC, Hanson RW. The key role of anaplerosis and cataplerosis for citric acid cycle function. $\mathrm{J} \mathrm{Biol} \mathrm{Chem}$ 2002; 277:30409-30412.

79 Hillier S, Charnetzky WT. Glyoxylate bypass enzymes in Yersinia species and multiple forms of isocitrate lyase in Yersinia pestis. J Bacteriol 1981; 145:452-458.

80 Kondrashov FA, Koonin EV, Morgunov IG, Finogenova TV, Kondrashova MN. Evolution of glyoxylate cycle enzymes in Metazoa: evidence of multiple horizontal transfer events and pseudogene formation. Biol Direct 2006; 1:31.

81 Kornberg HL, Beevers H. A mechanism of conversion of fat to carbohydrate in castor beans. Nature 1957; 180:35-36.

82 McKinney JD, Höner zu Bentrup K, Muñoz-Elías EJ, et al. Persistence of Mycobacterium tuberculosis in macrophages and mice requires the glyoxylate shunt enzyme isocitrate lyase. Nature 2000; 406:735-738.

83 Michelucci A, Cordes T, Ghelfi J, et al. Immune-responsive gene 1 protein links metabolism to immunity by catalyzing itaconic acid production. Proc Natl Acad Sci USA 2013; 110: 7820-7825.

84 McFadden BA, Purohit S. Itaconate, an isocitrate lyase-directed inhibitor in Pseudomonas indigofera. J Bacteriol 1977; 131:136-144.

85 Rubic T, Lametschwandtner G, Jost S, et al. Triggering the succinate receptor GPR91 on dendritic cells enhances immunity. Nat Immunol 2008; 9:1261-1269.

86 Chouchani ET, Pell VR, Gaude E, et al. Ischaemic accumulation of succinate controls reperfusion injury through mitochondrial ROS. Nature 2014; 515:431-435.
87 Stein M, Keshav S, Harris N, Gordon S. Interleukin 4 potently enhances murine macrophage mannose receptor activity: a marker of alternative immunologic macrophage activation. $J$ Exp Med 1992; 176:287-292.

88 Doyle AG, Herbein G, Montaner LJ, et al. Interleukin-13 alters the activation state of murine macrophages in vitro: comparison with interleukin-4 and interferon-gamma. Eur $J$ Immunol 1994; 24:1441-1445.

89 Mosser DM, Edwards JP. Exploring the full spectrum of macrophage activation. Nat Rev Immunol 2008; 8:958-969.

90 Biswas SK, Mantovani A. Macrophage plasticity and interaction with lymphocyte subsets: cancer as a paradigm. Nat Immunol 2010; 11:889-896.

91 Anthony RM, Urban Jr JF, Alem F, et al. Memory T(H)2 cells induce alternatively activated macrophages to mediate protection against nematode parasites. Nat Med 2006; 12:955-960.

92 Reece JJ, Siracusa MC, Scott AL. Innate immune responses to lung-stage helminth infection induce alternatively activated alveolar macrophages. Infect Immun 2006; 74:4970-4981.

93 Hesse M, Modolell M, La Flamme AC, et al. Differential regulation of nitric oxide synthase- 2 and arginase- 1 by type $1 /$ type 2 cytokines in vivo: granulomatous pathology is shaped by the pattern of L-arginine metabolism. J Immunol 2001; 167:6533-6544.

94 Mills CD, Kincaid K, Alt JM, Heilman MJ, Hill AM. M-1/ M-2 macrophages and the Th1/Th2 paradigm. J Immunol 2000; 164:6166-6173.

95 Pesce JT, Ramalingam TR, Mentink-Kane MM, et al. Arginase-1-expressing macrophages suppress Th2 cytokine-driven inflammation and fibrosis. PLoS Pathog 2009; 5:e1000371.

96 El Kasmi KC, Qualls JE, Pesce JT, et al. Toll-like receptor-induced arginase 1 in macrophages thwarts effective immunity against intracellular pathogens. Nat Immunol 2008; 9:13991406.

97 Pawlus MR, Hu CJ. Enhanceosomes as integrators of hypoxia inducible factor (HIF) and other transcription factors in the hypoxic transcriptional response. Cell Signal 2013; 25:18951903.

98 Imtiyaz HZ, Williams EP, Hickey MM, et al. Hypoxia-inducible factor 2alpha regulates macrophage function in mouse models of acute and tumor inflammation. J Clin Invest 2010; 120:2699-2714.

99 Szanto A, Balint BL, Nagy ZS, et al. STAT6 transcription factor is a facilitator of the nuclear receptor PPARgamma-regulated gene expression in macrophages and dendritic cells. Immunity 2010; 33:699-712.

100 Odegaard JI, Ricardo-Gonzalez RR, Goforth MH, et al. Macrophage-specific PPARgamma controls alternative activation and improves insulin resistance. Nature 2007; 447:1116-1120.

101 Nath N, Khan M, Paintlia MK, Singh I, Hoda MN, Giri S. Metformin attenuated the autoimmune disease of the central nervous system in animal models of multiple sclerosis. $J$ Immunol 2009; 182:8005-8014.

102 Gautier EL, Ivanov S, Williams JW, et al. Gata6 regulates aspartoacylase expression in resident peritoneal macrophages and controls their survival. $J$ Exp Med 2014; 211:1525-1531.

103 Bendtzen K, Mandrup-Poulsen T, Nerup J, Nielsen JH, Dinarello CA, Svenson M. Cytotoxicity of human pI 7 interleukin-1 for pancreatic islets of Langerhans. Science 1986; 
232:1545-1547.

104 Westwell-Roper CY, Ehses JA, Verchere CB. Resident macrophages mediate islet amyloid polypeptide-induced islet IL-1beta production and beta-cell dysfunction. Diabetes 2014; 63:1698-1711.

105 Larsen CM, Faulenbach M, Vaag A, et al. Interleukin-1-receptor antagonist in type 2 diabetes mellitus. $N$ Engl $J$ Med 2007; 356:1517-1526.

106 Donath MY. Targeting inflammation in the treatment of type 2 diabetes: time to start. Nat Rev Drug Discov 2014; 13:465476.

107 Nath N, Giri S, Prasad R, Salem ML, Singh AK, Singh I. 5-aminoimidazole-4-carboxamide ribonucleoside: a novel immunomodulator with therapeutic efficacy in experimental autoimmune encephalomyelitis. J Immunol 2005; 175:566574.

108 Ooi M, Nishiumi S, Yoshie T, et al. GC/MS-based profiling of amino acids and TCA cycle-related molecules in ulcerative colitis. Inflamm Res 2011; 60:831-840.

109 Weinert BT, Schölz C, Wagner SA, et al. Lysine succinylation is a frequently occurring modification in prokaryotes and eukaryotes and extensively overlaps with acetylation. Cell Rep 2013; 4:842-851.

110 Zhang Z, Tan M, Xie Z, Dai L, Chen Y, Zhao Y. Identification of lysine succinylation as a new post-translational modification. Nat Chem Biol 2011; 7:58-63.

111 Tan M, Peng C, Anderson KA, et al. Lysine glutarylation is a protein posttranslational modification regulated by SIRT5. Cell Metab 2014; 19:605-617.

(c) (i) (3) $\odot$ This work is licensed under the Creative Commons Attribution-NonCommercial-No Derivative Works 3.0 Unported License. To view a copy of this license, visit http:// creativecommons.org/licenses/by-nc-nd/3.0 